\title{
Erratum to: On the reliability and availability of replicated and rejuvenating systems under stealth attacks and intrusions
}

\author{
Luís Teixeira d'Aguiar Norton Brandão • \\ Alysson Neves Bessani
}

Published online: 12 May 2012

(C) The Brazilian Computer Society 2012

\section{Erratum to: J Braz Comput Soc (2012) 18:61-80 DOI 10.1007/s13173-012-0062-x}

The following is a list of corrections to several typos found in the original online version of [1]:

- in the caption of Fig. 10 (p. 75, right column) the correct analytic expression used to draw the reference curve $(e, \Delta)$ was $\left(1-e^{-r}\right) / r$, rather than $\left(1-e^{r}\right) / r$;

- Equation (10) (p. 79, left column) should be

$$
\begin{aligned}
& (\forall t>0, \lambda>0)\left(\forall n^{\prime}>n>f \geq 0\right)\left(\forall f^{\prime}>f: n^{\prime}>f^{\prime}\right) \\
& \left(\mathcal{R}_{n^{\prime}, f}^{\|, \lambda}(t)<\mathcal{R}_{n, f}^{\|, \lambda}(t)\right) \wedge\left(\mathcal{R}_{n^{\prime}, f}^{\|, \lambda}(t)<\mathcal{R}_{n^{\prime}, f^{\prime}}^{\|, \lambda}(t)\right)
\end{aligned}
$$

- Equation (14) (p. 79, left column) is valid for $t>0$.
The online version of the original article can be found under doi:10.1007/s13173-012-0062-x.

\section{Teixeira d'Aguiar Norton Brandão ( $\square)$} Electrical \& Computer Engineering Department, Carnegie Mellon University, 4720 Forbes Ave, CyLab, Collaborative Innovation Center, Pittsburgh, PA 15213, USA e-mail: luis.papers@gmail.com

L. Teixeira d'Aguiar Norton Brandão e-mail: lbrandao@cmu.edu

L. Teixeira d'Aguiar Norton Brandão · A. Neves Bessani LaSIGE, Faculdade de Ciências, Departamento de Informática, Universidade de Lisboa, Edifício C6, Piso 3, Campo Grande, 1749-016, Lisboa, Portugal

L. Teixeira d'Aguiar Norton Brandão e-mail: 1brandao@di.fc.ul.pt

A. Neves Bessani e-mail: bessani@di.fc.ul.pt

\section{References}

1. Brandão LTAN, Bessani AN (2012) On the reliability and availability of replicated and rejuvenating systems under stealth attacks and intrusions. J Braz Comput Soc 18:61-80. doi:10.1007/s13173012-0062-x 